\title{
Allogeneic CD25/Treg-depleted Donor Lymphocytes
}

National Cancer Institute

\section{Source}

National Cancer Institute. Allogeneic CD25/Treg-depleted Donor Lymphocytes. NCI

Thesaurus. Code C160729.

A preparation of allogeneic peripheral blood mononuclear cells (PBMCs) derived from an 8/8 HLA-matched donor that have been selectively depleted of CD4+CD25hiFoxp3+ regulatory T-cells (Tregs; CD25hi Tregs) with potential to enhance graft-versus-tumor (GVT) immune responses in patients with myeloid relapse following a hematopoietic stem cell transplant (HSCT) from a matched donor. Following collection and ex-vivo depletion of CD25hi Tregs from PBMCs derived from the original donor, the CD25/T regdepleted donor lymphocytes are infused into the patient. Upon administration, the CD25/T reg-depleted donor lymphocyte infusion (DLI) may enhance the proliferation of CD4+ and CD8+ effector T-cells (T effs) and potentially induce curative GVT immune responses in patients with myeloid relapse following HSCT. 